\title{
A Stable Algorithm for Computing the Inverse Error Function in the "Tail-End" Region
}

\author{
By Henry E. Fettis
}

Abstract. An iterative algorithm, simple enough to be executed on a desk top automatic computer, is given for computing the inverse of the function $x=\operatorname{erfc}(y)$ for small values of $x$.

In the present note, a simple method is proposed for computing values of $y$ for which the function

$$
\operatorname{erfc}(y)=\frac{2}{\pi^{1 / 2}} \int_{y}^{\infty} e^{-\eta^{2}} d \eta
$$

assumes a prescribed value $x$. This problem occurs in statistics, and also in many problems relating to heat transfer and diffusion. The last mentioned application led Philip [1] to consider the following function

$$
y=\operatorname{inverfc}(x)
$$

and methods for computing it. Later, Strecok [2] gave a more detailed treatment' and obtained power series expansions and representations in terms of Chebyshev polynomials.

When $x$ is close to unity, the inverted power series for $\operatorname{erf}(y)=1-x$ may be used to advantage. Strecok (loc. cit.) gives the first 200 terms, which may be found from a simple recurrence relation, as well as economized series of Chebyshev polynomials derived from the power series. The series will yield about 20 correct decimal places for $x>.125$. For smaller values of $x$, a new function

$$
R(x)=\text { inverfc }(1-x) /\left[-\ln \left(2 x-x^{2}\right)\right]^{1 / 2}
$$

is introduced which, in turn, can be expressed, in various intervals of $x$, by economized series.

In the present note, a simpler method is proposed to handle the region of small $x$. It is based on the representation of $\operatorname{erfc}(y)$ as a continued fraction [3]:

$$
\sqrt{ } \pi e^{-y^{2}} \operatorname{erfc}(y)=\frac{t}{1+\frac{\left(t^{2} / 2\right)}{1+\frac{2\left(t^{2} / 2\right)}{1+\frac{3\left(t^{2} / 2\right)}{1+\cdots+}}}}=G(t)
$$

where $t=1 / y$. Writing $F(t, x)=G(t) / \pi^{1 / 2} x$, we obtain the relation

Received July 2, 1973.

AMS (MOS) subject classifications (1970). Primary 33A20.

Key words and phrases. Inverse error function, inverse probability integral, error function, probability integral. 


$$
y^{2}=\ln F(x, y)
$$

which may be solved iteratively as

$$
y_{n+1}=\left[\ln F\left(x, y_{n}\right)\right]^{1 / 2} .
$$

As a starting value, Philip's approximation

$$
y \cong\left\{-\ln \left[\pi^{1 / 2} x(-\ln x)^{1 / 2}\right]\right\}^{1 / 2}
$$

may be used.

The above algorithm works best for small values of $x$. For larger values, the inverted power series proves to be more economical. One attractive feature of the present algorithm is that it may be used for all values of $x$ below a certain value, and does not require subdividing this region. Another feature is that it is simple enough to be executed directly on a desk top automatic computer (such as the HewlettPackard 9100).

Numerical experiments with the present method indicate that the power series requires more arithmetical operations when $x<.01$. The following table lists the comparison in the case where 12 figures of accuracy are required.

$\begin{array}{cccc}x & \begin{array}{c}\text { Number of terms } \\ \text { of power series }\end{array} & \begin{array}{c}\text { Number of terms } \\ \text { of continued fraction }\end{array} & \begin{array}{c}\text { Number of Iterations } \\ 1 \times 10^{-6}\end{array} \\ \text { Prohibitive } & 29 & 7 \\ 1 \times 10^{-4} & \text { Prohibitive } & 33 & 8 \\ 1 \times 10^{-2} & 949 & 51 & 11 \\ 5 \times 10^{-2} & 202 & 74 & 14 \\ .1 & 102 & 98 & 16 \\ .2 & 50 & 150 & 21 \\ .3 & 32 & 220 & 27 \\ .4 & 23 & 323 & 52 \\ .5 & 17 & 492 & 179 \\ .6 & 13 & \text { Prohibitive } & \text { Prohibitive } \\ .7 & 10 & \text { Prohibitive } & \text { Prohibitive } \\ .8 & 8 & \text { Prohibitive } & \text { Prohibitive } \\ .9 & 5 & \text { Prohibitive } & \text { Prohibitive }\end{array}$

Recalculation of Philip's table [1] by the present method* indicates that the following corrections should be made:**

$$
\text { For } \begin{aligned}
x & =10^{-5}, \quad y=3.1234132743, \\
x & =10^{-4}, \quad y=2.7510639057 .
\end{aligned}
$$

The corresponding values of $B(\theta)=2 / \pi^{1 / 2} e^{-y^{2}}$ should be appropriately corrected.

$$
\text { For } \begin{aligned}
x & =10^{-6}, & B & =7.186679956 \times 10^{-6}, \\
x & =10^{-5}, & B & =6.540392772 \times 10^{-5}, \\
x & =10^{-4}, & B & =5.828560144 \times 10^{-4}, \\
x & =.05, & B & =.1653076207 .
\end{aligned}
$$

* Calculations made by James C. Caslin on the CDC 6600.

** Philip's " $\theta$ " corresponds to " $x$ " in the present paper. 
Aerospace Research Laboratories Wright-Patterson Air Force Base Ohio 45433

1. J. R. PHIII, "The function inverfe $\theta$," Austral. J. Phys., v. 13, 1960, pp. 13-20. MR 22 \#9626.

2. A. J. Strecox, "On the calculation of the inverse of the error function," Math. Comp., v. 22,1968 , pp. 144-158, MR 36 \#119.

3. H. S. WALL, Analytic Theory of Continued Fractions, Van Nostrand, New York, 1948, p. 358. MR 10, 32. 